\title{
Acoustic Emission Waveform Changes For Varying Seeded Defect Sizes
}

\author{
Saad Al-Dossary ${ }^{1}$, R.I. Raja Hamzah², D. Mba ${ }^{2}$ \\ ${ }^{1}$ Consulting services, Saudi Aramco, Dhahran, Saudi Arabia \\ ${ }^{2}$ School of Mechanical Engineering, Cranfield University, Cranfield, Beds. MK43 OAL. \\ E-mail: r.i.raja-hamzah-2003@cranfield.ac.uk.
}

Keywords: Acoustic emissions, bearing defect size, bearing fault diagnosis, condition monitoring.

\begin{abstract}
The investigation reported in this paper was centered on the application of the Acoustic Emissions (AE) technology for characterising the defect sizes on a radially loaded bearing. The aim of this investigation was to ascertain the relationship between the duration of $\mathrm{AE}$ transient bursts associated with seeded defects to the actual geometric size of the defect. It is concluded that the geometric defect size can be determined from the AE waveform.
\end{abstract}

\section{Introduction}

Acoustic emissions (AE) are defined as the range of phenomena that results in structure-borne propagating waves being generated by the rapid release of energy from localised sources within and/or, on the surface of a material. In this particular investigation, AE's are defined as the transient elastic waves generated by the interaction of two surfaces in relative motion. The interaction of surface asperities and impingement of the bearing rollers over a seeded defect will generate AE's. Due to the high frequency content of the AE signatures typical mechanical noise (less than $20 \mathrm{kHz}$ ) is eliminated. A comprehensive review of the application of the AE technology for bearing monitoring was presented by (Mba and Rao, 2006) where it was shown that the AE technology offered earlier fault identification than vibration analysis. This investigation builds on the investigation of (Al-Ghamdi et al., 2004; Al-Ghamdi and Mba, 2005) by exploring further the relationship between time-domain $\mathrm{AE}$ waveform characteristics with seeded defect geometric dimensions of the outer race.

\section{Experimental apparatus and data acquisition}

The test bearing was fitted on a rig which consisted of a shaft that was driven by a motor. The shaft was supported by two large slave bearings, see figure 1. The test bearing was a split Cooper cylindrical roller type 01B40MEX, with a bore diameter of $40 \mathrm{~mm}$, external diameter of $84 \mathrm{~mm}$, pitch circle diameter of $166 \mathrm{~mm}$, roller diameter of $12 \mathrm{~mm}$, and 10 rollers in total. This bearing was selected due to its split design which would facilitate the assembling and disassembling of the bearing on the shaft after each test. 
The AE acquisition system consisted of a piezoelectric type sensor (Physical Acoustic Corporation type WD) fitted onto the top half of the bearing housing. The transducer had an operating frequency of 100 to $1000 \mathrm{kHz}$. The signal from the transducer was amplified at $40 \mathrm{~dB}$ and sampled at $8 \mathrm{MHz}$.

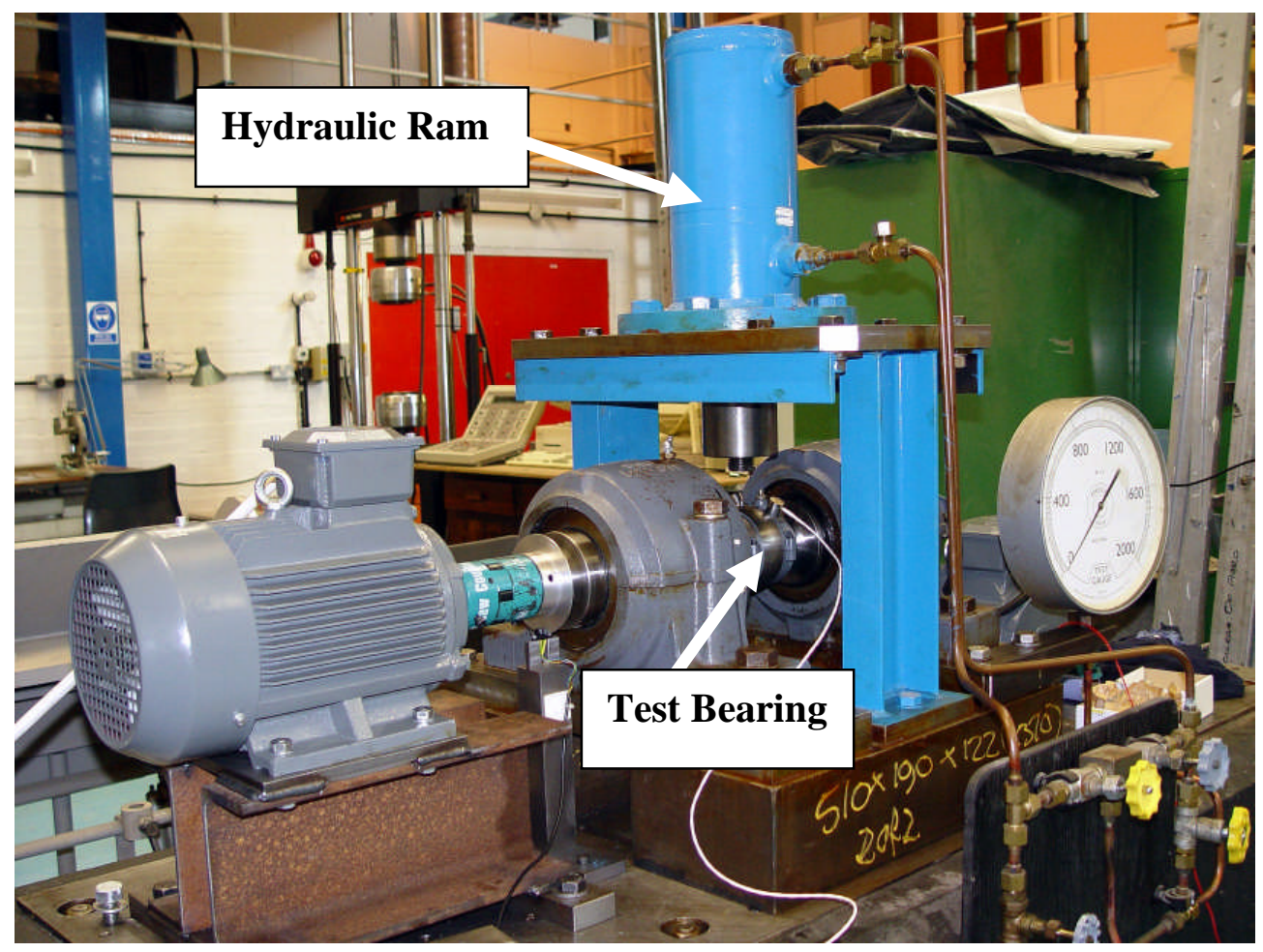

Fig. 1 Layout of experimental test-rig

\section{Experimental Procedure}

In an attempt to understand how the defect size influenced AE waveform an incremental procedure for simulating increasing defect sizes was established. This involved starting a defect sequence on a bearing with a point defect (D1) and increasing the length along the circumferential direction to a maximum value for a fixed width across the race. Once this maximum length was achieved the width of the defect was then expanded. The defects on the bearing elements were made by using an electric engraver with a carbide tip. A breakdown on the incremental defect procedure is detailed in table 1 . The defect sizes were measured by its Length $(\mathrm{mm})$ and Width $(\mathrm{mm})$, where the length was measured circumferentially in the direction of the rollers and the width is defined as the distance across the bearing race. Experimental tests were at 1500rpm for three load conditions; 2.7, 5.3, and $8 \mathrm{kN}$. 


\begin{tabular}{|c|c|}
\hline \multicolumn{2}{|c|}{ Bearing $\mathbf{1}(\boldsymbol{L} \boldsymbol{X} \mathbf{W}) \mathbf{m m}$} \\
\hline D1 & Circle $D=0.5 \mathrm{~mm}$ \\
\hline $\boldsymbol{D 2}$ & $0.9 \times 2.5 \mathrm{~mm}$ \\
\hline $\boldsymbol{D 3}$ & $0.9 \times 4$ \\
\hline $\boldsymbol{D 4}$ & $0.9 \times 8$ \\
\hline $\boldsymbol{D 5}$ & $0.9 \times 12$ \\
\hline $\boldsymbol{D 6}$ & $3 \times 12$ \\
\hline $\boldsymbol{D 7}$ & $5 \times 12$ \\
\hline $\boldsymbol{D} 8$ & $7 \times 12$ \\
\hline $\boldsymbol{D 9}$ & $9 \times 12$ \\
\hline
\end{tabular}

Table 1 Incremental defect sizes (outer race)

\section{Analysis}

The energy values were compared for increasing outer race defect sizes under varying and load conditions. It was noted that energy values increased with increasing defect size and increasing load, see figure 2. If the defects simulated were to produce AE transient bursts, as each rolling element passed the defect, it was envisaged that the AE bursts would be detected at a rate equivalent to the outer race defect frequencies (approximately 4-times rotational speed). The method of calculating the burst duration involved defining the start and end of each burst and determining the duration, as shown in figure 3. The duration value presented for each defect condition was an average value taken from over twenty AE bursts per defect size.

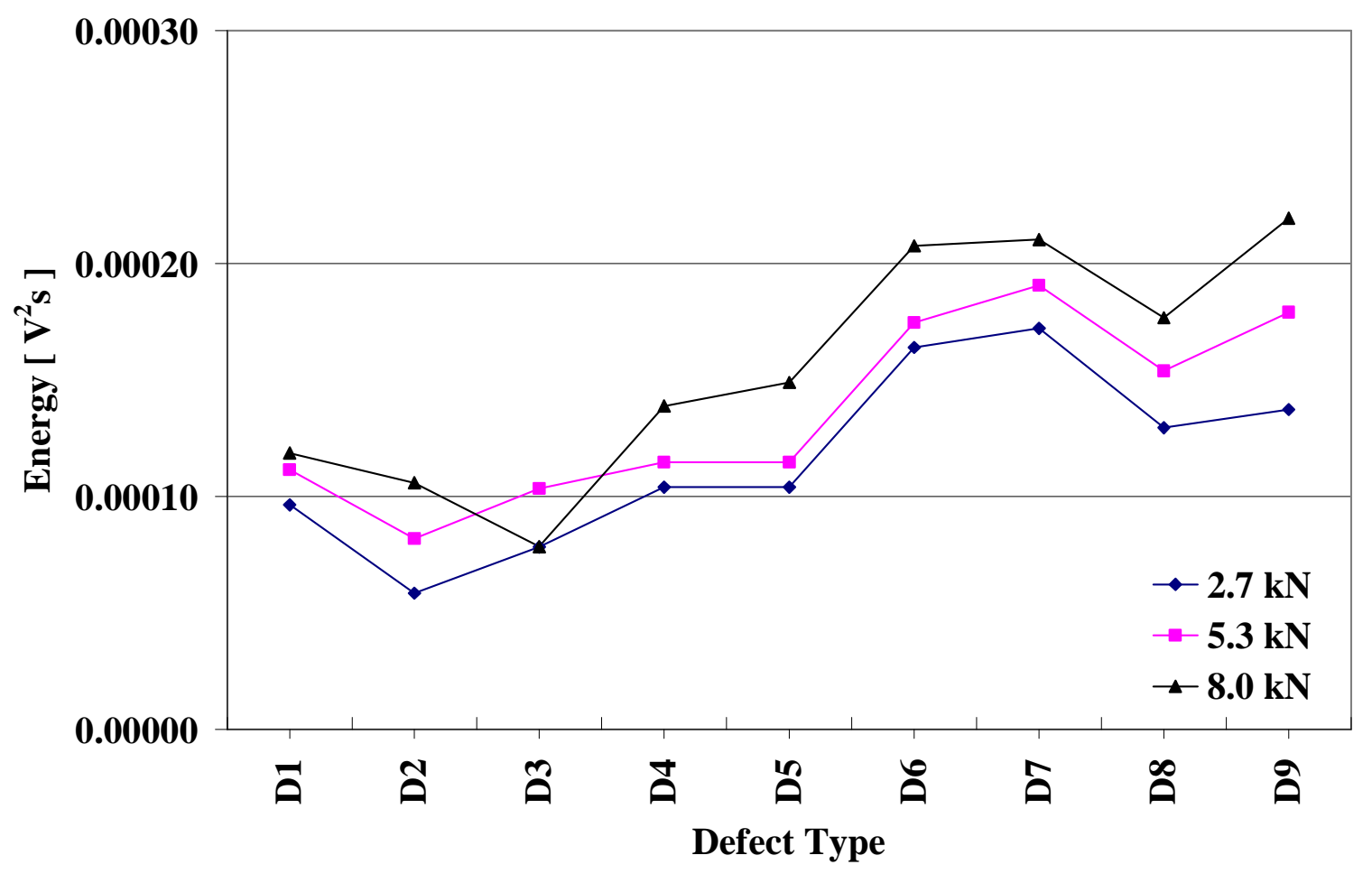

Fig. 2 AE Energy for varying defect and load conditions 


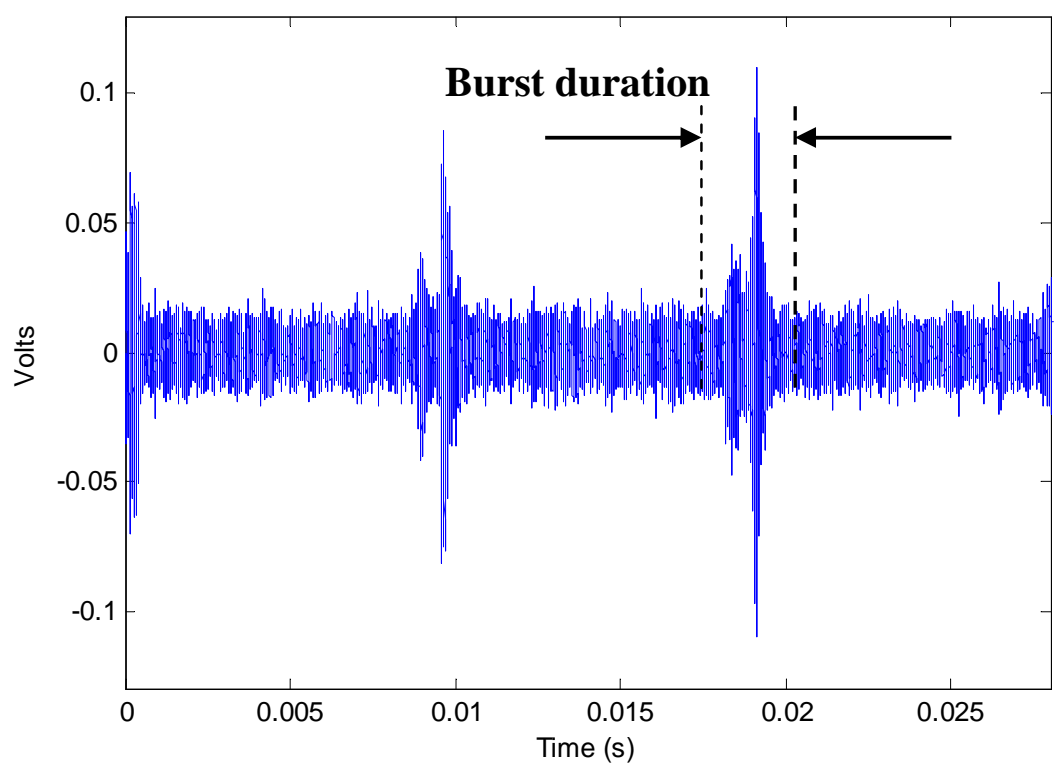

Fig. 3 Example procedures for determining the AE transient burst duration

It is important to note that defect D1 was a point defect, D2 to D5 had fixed length with increasing width and D6 to D9 had a fixed width with increasing length, as described in table 1. Figure 4 presents waveforms that offer the reader a qualitative observation of the influence of changing defect size on AE waveform.

For defect D1 no AE transient bursts were evident above the operational background noise levels. For defects D2 to D5 the AE burst duration associated with the defect condition remained relatively constant irrespective of the load condition, however, the duration of the AE burst associated with defects D6 to D9 increased with increasing defect size along the circumferential direction of the roller (length), see figure 5 . 


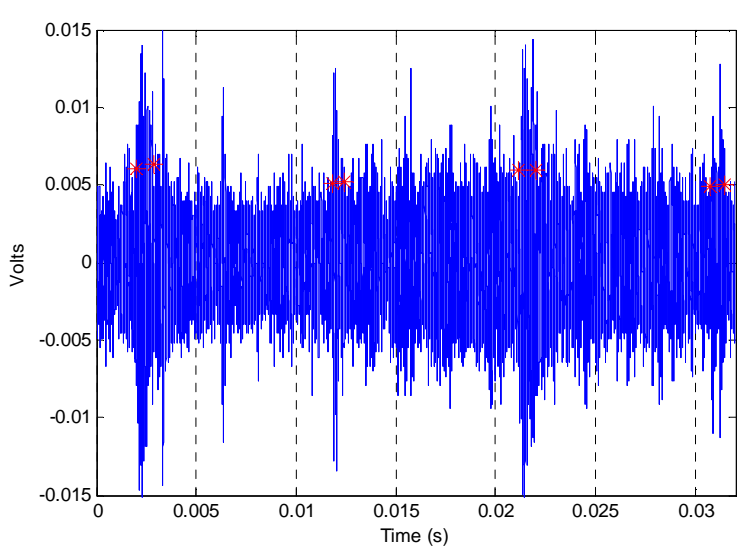

Defect D2

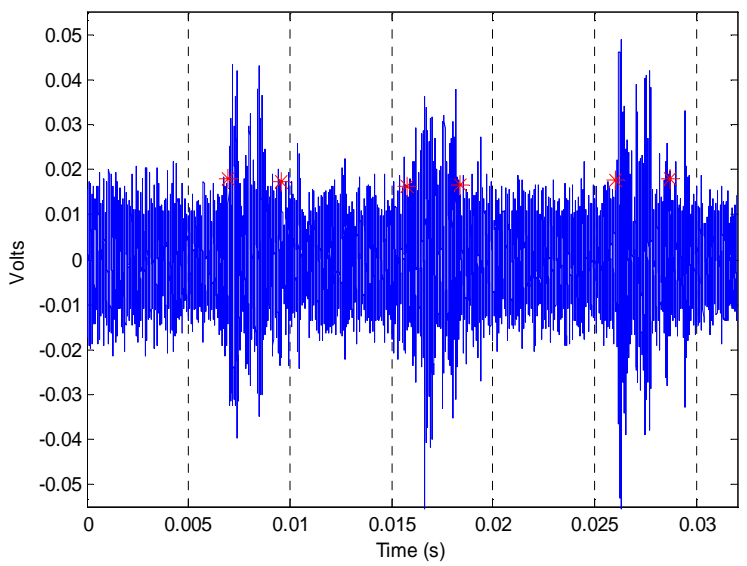

Defect D7

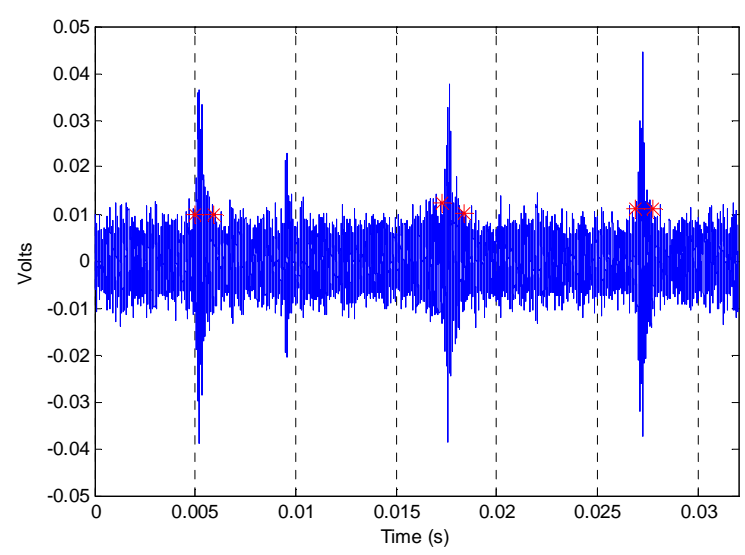

Defect D4

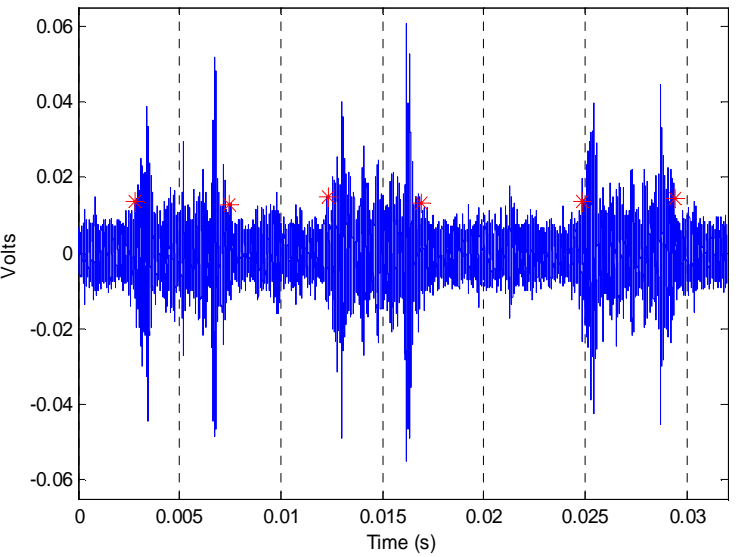

Defect D9

Fig. 4 Different AE waveforms for varying defect sizes 


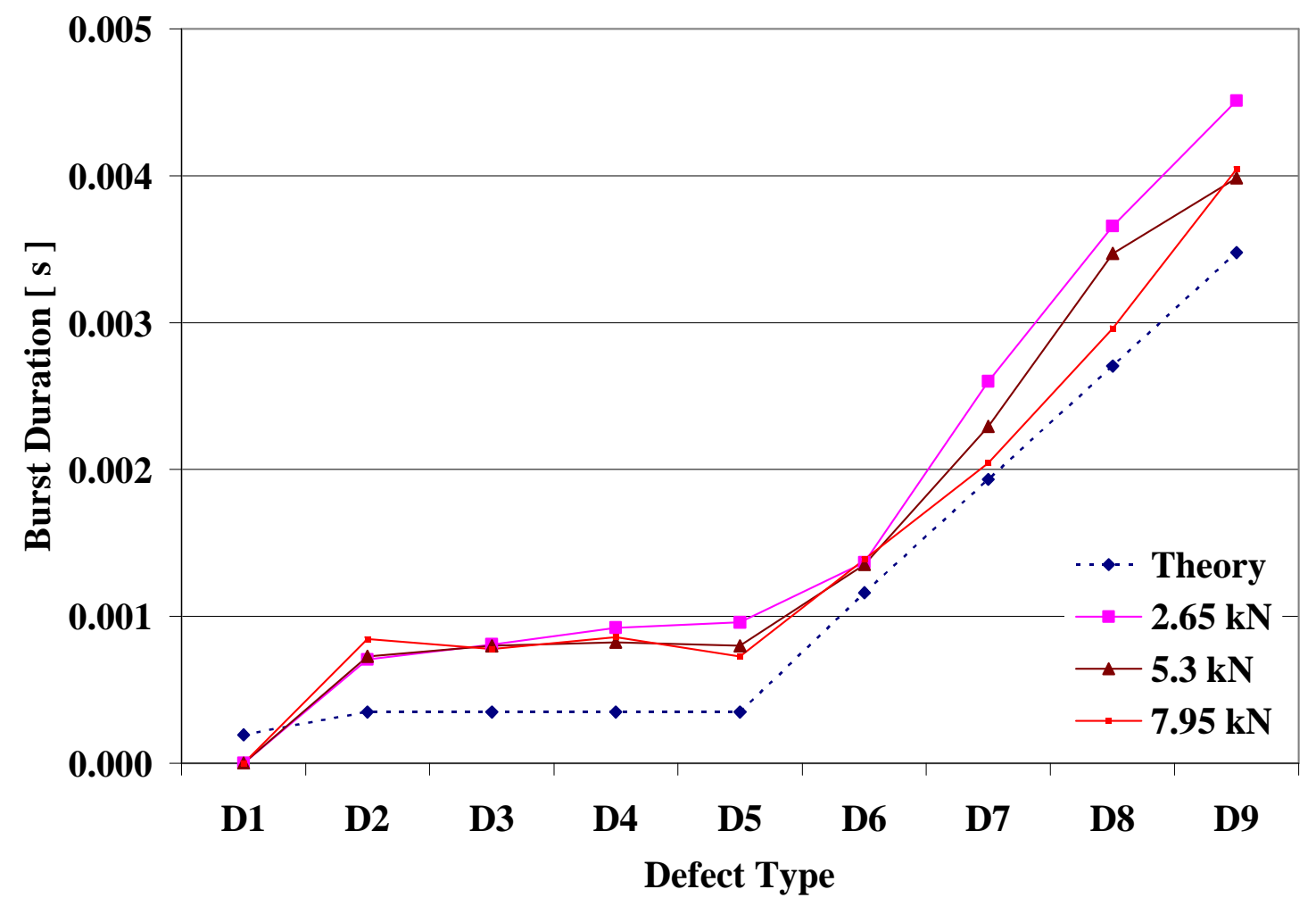

Fig. 5 Burst Duration at 1500rpm; outer race defect

The theoretical time duration of the roller passing over the defect was also calculated based on the rotational speed of the shaft and the relative velocities of the elements within the bearing. The objective was to correlate the theoretically determined time duration over which the roller passed the defect to the duration of $\mathrm{AE}$ transient burst associated with the specified defect. The result shows that experimental and theoretical values followed the same pattern, see figure 5 . However the experimental values were larger than the calculated theoretical values by as much as $170 \%$ for defects associated with increasing width. Variation with theoretical values for defects of increasing length was in the order of $30 \%$; see tables 2 . This suggested that the measured experimental burst duration was more representative of the defect size for conditions were the defect was in the circumferential direction (D6-D9). A reason for the difference between theoretical and experimental time durations is attributed to the decay characteristics of the AE transient bursts. The visual end of the $\mathrm{AE}$ transient employed for calculating the burst duration is not actually the instant in time when the generation of $\mathrm{AE}$ ceased as there is an exponential decay associated with $\mathrm{AE}$ transient bursts. To reduce the difference between the theoretical and experimental results will require employing much higher sampling rates to aid discrimination. 


\begin{tabular}{|c|c|c|c|c|c|c|c|}
\hline \multirow{2}{*}{ Defect } & Theory & \multicolumn{2}{|c|}{ Burst Duration (seconds) } & \multicolumn{2}{c|}{ \% Difference from theory } \\
\cline { 3 - 8 } & (Seconds) & $\mathbf{2 . 7} \mathbf{k N}$ & $\mathbf{5 . 3} \mathbf{~ k N}$ & $\mathbf{8 ~ k N}$ & $\mathbf{2 . 7} \mathbf{~ k N}$ & $\mathbf{5 . 3} \mathbf{~ k N}$ & $\mathbf{8 ~ k N}$ \\
\hline D1 & $1.93 \mathrm{E}-04$ & $0.00 \mathrm{E}+00$ & $0.00 \mathrm{E}+00$ & $0.00 \mathrm{E}+00$ & $0 \%$ & $0 \%$ & $0 \%$ \\
\hline D2 & $3.48 \mathrm{E}-04$ & $7.06 \mathrm{E}-04$ & $7.27 \mathrm{E}-04$ & $8.44 \mathrm{E}-04$ & $103.0 \%$ & $109.2 \%$ & $142.8 \%$ \\
\hline D3 & $3.48 \mathrm{E}-04$ & $8.07 \mathrm{E}-04$ & $8.00 \mathrm{E}-04$ & $7.78 \mathrm{E}-04$ & $132.3 \%$ & $130.1 \%$ & $123.9 \%$ \\
\hline D4 & $3.48 \mathrm{E}-04$ & $9.24 \mathrm{E}-04$ & $8.22 \mathrm{E}-04$ & $8.58 \mathrm{E}-04$ & $165.7 \%$ & $136.5 \%$ & $147.0 \%$ \\
\hline D5 & $3.48 \mathrm{E}-04$ & $9.60 \mathrm{E}-04$ & $8.00 \mathrm{E}-04$ & $7.27 \mathrm{E}-04$ & $176.2 \%$ & $130.3 \%$ & $109.3 \%$ \\
\hline D6 & $1.16 \mathrm{E}-03$ & $1.37 \mathrm{E}-03$ & $1.35 \mathrm{E}-03$ & $1.39 \mathrm{E}-03$ & $18.0 \%$ & $16.8 \%$ & $19.9 \%$ \\
\hline D7 & $1.93 \mathrm{E}-03$ & $2.60 \mathrm{E}-03$ & $2.29 \mathrm{E}-03$ & $2.04 \mathrm{E}-03$ & $34.6 \%$ & $18.7 \%$ & $5.8 \%$ \\
\hline D8 & $2.70 \mathrm{E}-03$ & $3.66 \mathrm{E}-03$ & $3.47 \mathrm{E}-03$ & $2.96 \mathrm{E}-03$ & $35.3 \%$ & $28.3 \%$ & $9.5 \%$ \\
\hline D9 & $3.48 \mathrm{E}-03$ & $4.51 \mathrm{E}-03$ & $3.99 \mathrm{E}-03$ & $4.04 \mathrm{E}-03$ & $29.7 \%$ & $14.7 \%$ & $16.3 \%$ \\
\hline
\end{tabular}

Table 2 Experimental and theoretical AE burst duration (Outer race)

\section{Conclusions}

An increase in defect size resulted in an increase in levels of AE energy. In conclusion, the measurement of $\mathrm{AE}$ energy over a duration equivalent to one rotation of the shaft has been shown to offer an indication of increasing defect severity for outer race defects. A correlation between the geometric size of outer race defects and the AE burst duration associated with such defects has been shown. This is a significant finding which in the longer term, and with further research, offers opportunities for prognosis.

\section{References}

1. Abdullah M. Al-Ghamdi, P. Cole, Rafael Such, D. Mba. (2004), "Estimation of bearing defect size with Acoustic Emission”, INSIGHT, Vol. 46, No. 12, pp. 758-761.

2. Abdullah M. Al-Ghamdi and D. Mba. (2005) "A comparative experimental study on the use of Acoustic Emission and vibration analysis for bearing defect identification and estimation of defect size", Mechanical Systems and Signal Processing, Accepted MSSP04-98R2.

3. D. Mba \& Raj B.K.N. Rao. (2006), "Development of Acoustic Emission Technology for Condition Monitoring and Diagnosis of Rotating Machines; Bearings, Pumps, Gearboxes, Engines and Rotating Structures", The Shock and Vibration digest, 38/1, pp. 3-16. 